\title{
Neurología de la resiliencia y desastres
}

\author{
Neurology of resilience and disasters
}

\section{Rolando J. Naranjo Álvarez}

Máster en Psiquiatría Social. Especialista de II Grado en Neurología. Policlínico de Especialidades "Julio A. Mella". Guanabacoa. La Habana, Cuba.

\section{RESUMEN}

Con el propósito de explicar las generalidades sobre la neurología de la resiliencia frente a los desastres que pueden afectar a las comunidades, se describen los aspectos psíquicos vinculados a los grandes contratiempos que pueden dañar a cualquier colectividad. Desde una mirada a las necesidades humanas según el grado de perentoriedad, se realiza una aproximación a los desastres naturales y se exponen las bases neurales, estructurales y neurobioquímicas que subyacen en los mecanismos de la imprescindible tenacidad cognitiva, nombrada como resiliencia humana individual y colectiva. Queda revisada la evolución del concepto, las manifestaciones clínicas más importantes y la conducta a seguir muy consustancial al nivel de atención primaria de salud.

Palabras clave: Resiliencia, desastres, bases neurales, atención primaria de salud, tenacidad cognitiva.

\begin{abstract}
With the objective of explaining the general aspects of neurology of resilience in disasters that may affect the communities, this paper describes the psychical aspects related to big setbacks that may affect any community. Taking the human needs into account according to their degree of urgency, the paper approached to natural disasters and disclosed the neural, structural and neurochemical underlying bases in the mechanisms of the indispensable cognitive tenacity called individual
\end{abstract}


and collective human resilience. The evolution of this concept, the most important clinical manifestations and the behaviour to be adopted at the primary health care level was reviewed.

Key words: Resilience, disasters, neural basis, primary health care, cognitive tenacity.

\section{INTRODUCCIÓN}

Del psiquismo, afectividad y necesidades humanas se conoce que:

- Los fenómenos psíquicos y procesos mentales son inseparables de la esencia del ser humano y toda persona desempeña o protagoniza una función más o menos variable en el entorno donde transcurre su existencia. Desde el punto de vista ecológico, la vida colectiva modifica el medio ambiente y a la vez, la sociedad se integra y forma parte de dicho medio. Entonces, cualquier alteración importante del proceso de salud de un individuo tiene al menos, la capacidad de trascender a lo social.

- La calidad y cualidades del funcionamiento mental en cuanto a la esfera afectiva humana con su conjunto de instintos, necesidades, motivaciones y emociones, expresan la relación que se establece de manera dinámica, entre las necesidades humanas y el grado de satisfacción que se alcanza sin deslindarse del medio donde se desenvuelven y menos aún de las agresiones a que son sometidas.

- Las necesidades humanas surgen y mantienen un determinado orden de prioridad ya sean más o menos sentidas. En el recuadro 1 se muestran sus niveles y ejemplos de mayor interés:

\begin{tabular}{|c|c|c|}
\hline Nivel de necesidad & Necesidades & No satisfechas producen \\
\hline $\begin{array}{l}\text { I. Biológico (primitivas). } \\
\text { Demandas vitales }\end{array}$ & $\begin{array}{l}\text { Alimentación, defensa, } \\
\text { integridad, procreación. }\end{array}$ & $\begin{array}{l}\text { Muerte del individuo o de } \\
\text { la especie. }\end{array}$ \\
\hline II. Psicológico & $\begin{array}{l}\text { Estimulación, seguridad y } \\
\text { amor. }\end{array}$ & $\begin{array}{l}\text { Trastomo mental. } \\
\text { (Ej. Psicosis } \\
\text { experimental). }\end{array}$ \\
\hline III. Social & $\begin{array}{l}\text { Comunicación, } \\
\text { comprensión, aceptación, } \\
\text { trato amable y } \\
\text { reconocimiento. }\end{array}$ & $\begin{array}{l}\text { Hostilidad, marginación y } \\
\text { disminución de la } \\
\text { autoestima. }\end{array}$ \\
\hline IV. Cultural & $\begin{array}{l}\text { Legítimos intereses } \\
\text { personales. }\end{array}$ & $\begin{array}{l}\text { Frustración, } \\
\text { desmotivación. }\end{array}$ \\
\hline V. Espiritual (desarrolladas) & $\begin{array}{l}\text { Los valores: Ejs. empatía, } \\
\text { compasión, solidaridad. }\end{array}$ & $\begin{array}{l}\text { Frustración de la vocación } \\
\text { de servicio (laboral, } \\
\text { profesional, reliqioso). }\end{array}$ \\
\hline
\end{tabular}


En todos los niveles se puede encontrar afectación personal o grupal cuando ocurren grandes eventos como son los desastres y en estas circunstancias ganan enorme valor las características personales y por extensión, colectivas, que permiten afrontar los hechos y erradicarlos. A la vez, la realidad circundante según el escenario donde suceda el evento desafortunado se torna determinante.

A medio camino transitado desde la formulación de las metas del tercer milenio hasta el año 2015, es lamentable que no se hayan solucionado casi ninguno de los propósitos redactados por el organismo competente de la ONU. ${ }^{2}$ Los de mayor relevancia indican que no se ha erradicado la pobreza, no se logra la educación a nivel mundial y mucho menos se procura el impostergable sostenimiento del medio ambiente, precisamente los más vinculados a la aparición de eventos con probabilidades de convertirse en verdaderas catástrofes.

Esta verdad nada ilusoria obliga a preparar los recursos humanos requeridos para enfrentar los desastres y conocer acerca de la resiliencia se vuelve sumamente conveniente.

\section{RESILIENCIA. EVOLUCIÓN DEL CONCEPTO A TENACIDAD COGNITIVA}

La palabra resiliencia se tomó prestada de la física, de donde su precursor en emplearla en psicología, el paidopsiquiatra conductista Michael Rutter, la reduce a "cierta flexibilidad social para la adaptación". Posteriormente, la definición trascendió el conductismo, cuando el etólogo Boris Cyrulnik estudia a personas sobrevivientes de campos de concentración y niños, tanto de orfanatorios como los llamados "de la calle." 3

Se entiende por resiliencia a la capacidad psicológica de las personas, para sobreponerse a episodios de dolor emocional o grandes contratiempos y académicamente, se define la resiliencia psíquica como el "resultado de múltiples procesos mentales que contrarrestan las situaciones nocivas."

El término en castellano no tiene la connotación adecuada, por lo que se prefiere llamar a esa importante cualidad humana como Tenacidad Cognitiva (TC).

\section{Bases neurales y neurobioquímicas de la resiliencia o tenacidad cognitiva}

Se conocen las bases neurobiológicas y neurobioquímicas que subyacen como fundamentos de los cambios mentales que acompañan a la conducta resiliente:

- Están vinculadas con la retroacción de vivencias marcadamente estresantes. Diversas regiones cerebrales relacionadas con circuitos neuronales que conforman bases neurales estructurales y funcionales de la memoria y la vigilia, los cuales se reactivan de manera autónoma, para sustentar los recuerdos. Interviene la neocorteza cerebral y a nivel subcortical, el complejo amigdalino, el hipocampo y el locus cerúleo.

- De la neuroquímica que participa en la resiliencia se han precisado algunas de las sustancias hormonales y neurotransmisoras del importante eje cerebro-hipotálamosuprarrenal-gonadal que favorecen o perjudican y tienen un particular interés por ser susceptibles de medición y análisis: 
El cortisol, relacionado con los estados de alerta, vigilia y atención focalizada, atenta contra el comportamiento resiliente cuando se encuentra en concentraciones elevadas en el organismo. El exceso de cortisol perjudica el desarrollo normal, la reproducción y la respuesta inmunológica.

La testosterona es muy afectada por los procesos de estrés. Las situaciones de sufrimiento psicológico se acompañan de bajos índices de testosterona en sujetos de ambos sexos y esto provoca una reducción de la capacidad atencional, disminución de la proactividad y merma de la autoconfianza, junto a pobre asertividad, estados de ánimo con predominio de la depresión con dificultad para ejercer el pensamiento colateral, paralelo a disfunciones sexuales y poca creatividad.

La di-hidro-epi-androsterona (DHEA) tiene la cualidad de inhibir las sobreexpresiones de glutamato y cortisol (glucocorticoides en general), además de disminuir la actividad del colesterol, por lo que es proresiliente de forma directa e indirecta al servir de mecanismo también preventivo de afecciones cardiocirculatorias y cerebrovasculares.

La galanina protege contra la isquemia a la mayoría de los sustratos anatomofuncionales involucrados con la resiliencia. Se produce en el intestino y al distribuirse mediante el sistema circulatorio, alcanza el encéfalo con preferencia por el complejo amigdalino, el hipocampo, el locus cerúleo y la zona prefrontal de ambos lóbulos cerebrales.

\section{Sintomatología relativa a la Resiliencia o Tenacidad Cognitiva}

Para el estudio de las manifestaciones clínicas es útil la clasificación neurobiológica de las personas que categoriza a los individuos como:

- Personalidades poco o no resilientes / con pobre o ninguna TC.

- Personalidades pro-resilientes / son personas con TC.

- Gama de gradaciones intermedias.

Las personas poco o no resilientes tienen la característica de padecer frecuentes y muy intensos episodios de reactivación de la memoria conciente del momento estresante como pensamientos compulsivos e intrusivos.

Los sujetos pro-resilientes son capaces de sobreponerse y superar los momentos de dolor emocional o contratiempos e incluso, salir fortalecidos de la experiencia negativa con evidente firmeza de ánimo.

Mas, como suele ocurrir con las variables analógicas en psicología, no digitalmente cuantificables, existe un amplísimo abanico intermedio de posibilidades que se apartan del esquema cerrado de sujetos sí o no resilientes.

Estadísticamente, se observa con relativa frecuencia, aunque no generalizable que los individuos con mayor capacidad intelectual y volumen de conocimientos, al poder procesar mejor las situaciones traumatizantes y afrontar las agresiones estresantes, son más resilientes en lo emocional y a nivel celular neuronal. ${ }^{4}$

\section{TENACIDAD COGNITIVA (RESILIENCIA) Y DESASTRES}


Los eventos que constituyen desastres o catástrofes ponen a prueba la resiliencia individual y por extensión lógica, a las comunidades afectadas. Se puede considerar entonces la existencia de comunidades con mayor o menor resiliencia y cada una requerirá un tratamiento específico según las condiciones en que se formen.

\section{Definiciones y características de los desastres}

Se conoce como desastre al evento súbito, desbordante, provocado por la acción humana o por la naturaleza propiamente. Su ocurrencia afecta la unidad biopsicosocial, cultural y espiritual que son las personas.

Dada su envergadura, modifican negativamente los patrones cotidianos de existencia y generan reales crisis en las personas y comunidades. Por algo se afirma que el verdadero carácter humano de las personas se manifiesta en los desastres.

Los desastres, ya sean de pequeñas proporciones, medianos o grandes, por cuánto pueden repercutir sobre las personas y el medio, son capaces de afectar localidades, naciones, regiones continentales o extenderse a gran parte del planeta.

Además de los resultados nefastos que ocasionan las manifestaciones violentas, sin duda desastrosas, a grandes o medianas escalas como los conflictos bélicos o los enfrentamientos ideo-filosófico-religiosos, económicos o interétnicos, cualquiera sea el pretexto, los desastres se estudianclasificados de manera académica en: telúricotectónicos, meteorológicos, topológicos y biológicos. El extremo de mayor gravedad se entiende como catástrofe. El recuadro 2 muestra ejemplos de desastres de diferentes naturalezas.

Recuadro 2. Principales desastres o catástrofes que demandan tenacidad cognitiva (resiliencia)

\begin{tabular}{|l|l|}
\hline Naturaleza del desastre & Ejemplos de desastres \\
\hline Telúrico / Tectónico & Erupción volcánica, terremotos y tsunamis. \\
\hline Meteorológicos & $\begin{array}{l}\text { Sequía, ciclones, alteraciones notables de la } \\
\text { temperatura del aire. }\end{array}$ \\
\hline Topológicos & $\begin{array}{l}\text { Inundaciones, deslizamientos, aluviones, aludes y } \\
\text { derrumbes. }\end{array}$ \\
\hline Biológicos & Epidemias y pandemias infecciosas. \\
\hline Manifestaciones violentas & $\begin{array}{l}\text { Guerras, masacres, accidentes colectivos como } \\
\text { choques de trenes y otros. }\end{array}$ \\
\hline
\end{tabular}

\section{Proceso desastre-resiliencia o dinámica desastre-tenacidad cognitiva}

El desastre según evoluciona en el tiempo, ocasiona alteraciones en el funcionamiento psicológico que atraviesan varias fases. Al inicio genera incertidumbre creciente, aparecen los inevitables rumores y es indudable el grado de vulnerabilidad, a lo cual se suma una tensión que va en aumento. En el segundo 
momento, aparece francamente el miedo, la confusión y es muy probable la desorganización. Es la etapa en la cual son ya ostensibles las "necesidades" humanas.

Un tercer lapso es llamado poscrítico o crónico. Se caracteriza por ser palpable el estrés, el temor, la ofuscación, la labilidad emocional, actitudes de negación y pueden verse crisis dentro del seno de las familias. Durante esta fase no son raros los hechos vandálicos y de violencia en general.

Finalmente, la fase de recuperación en la cual se observan estados depresivos y cambios emocionales más duraderos. Aquí ya se habla de "estrés postraumático", aparecen manifestaciones de violencia intrafamiliar puertas adentro y con frecuencia, pasividad o conductas de acomodamiento.

El fenómeno resiliente consta de una dinámica que atraviesa varias momentos identificables con mayor claridad cuando se analiza a nivel de comunidad: acciones de defensa y protección, búsqueda del equilibrio que enfrenta a la tensión, compromiso y arrostre del desafío, la superación, la valoración de lo que significa, la actitud positiva de los sujetos, la toma de responsabilidad y la creatividad; todas las cuales se tienen en cuenta para ofrecer un tratamiento.

\section{FAVORECER LA TENACIDAD COGNITIVA}

La conducta o tratamiento auxiliador para favorecer la resiliencia en cuanto a desastres se enmarca en sus dos fases fundamentales: antes de la ocurrencia del evento, para el manejo integral de los riesgos mediante la promoción y la prevención específica de la probabilidad de ocurrencia de daños, así como la planificación de tareas, sobre todo de capacitación e información; y después de culminada la etapa de impacto del suceso desastroso, en la cual solo se espera rescate y salvamento o preservación de la vida, se comienza la aplicación de los métodos de rehabilitación y readaptación junto a las faenas de recuperación y reconstrucción.

Readaptar el funcionamiento individual y colectivo es en esencia, volver a la resiliencia o adquirirla quienes eran no resilientes. En todo momento están en juego las necesidades tanto primitivas como las más desarrolladas. Debe insistirse en el intento de satisfacer las necesidades y no obviar las de carácter espiritual. El hombre es un ser espiritual. La espiritualidad humana, religiosa o no, es una antigua y poderosa fuente de energía si es canalizada adecuadamente. ${ }^{5}$

Una vez sobrepasado el desastre, es muy oportuno ofrecer un espacio o escenario de expresión y para obtener los mejores resultados, hacer valer la intersectorialidad y buscar que se involucre la mayor parte de los miembros de la comunidad afectada. Individualmente, dar margen para la resignación desde una posición de respeto hacia las víctimas del enorme contratiempo a la vez que se instrumentan y facilitan las ayudas. El propósito final de la ayuda tiene que ser la activación de la resiliencia personal y grupal antes y después de un evento de desastre.

\section{REFERENCIAS BIBLIOGRÁFICAS}


1. González R. La unidad bio-psico-socio-cultural y espiritual del hombre. Su importancia en la formación del médico. Rev Hosp Psiq Habana [serie en Internet]. 2004[citado 2008]. Disponible en:

http://www.revistahph.sld.cu/hph0104/hph08104.htm

2. United Nations. The Millennium Development Goals Report. DPI/2539B - July 2009. New York: UN;2009.

3. Poseck V. Resistir y rehacerse: una reconceptualización de la experiencia traumática desde la psicología positiva. Revista de Psicología Positiva. 2004:1.

4. Poseck V, Carbelo B, Vecina Ma L. La experiencia traumática desde la psicología positiva: resiliencia y crecimiento postraumático. Papeles del Psicólogo. $2006 ; 27(1): 40-9$.

5. Fossarelli P. Medicine, spirituality, and patient care. JAMA. 2008;20:836-8.

Recibido: 12 de agosto de 2009.

Aprobado: 3 de febrero de 2010.

Rolando J. Naranjo Álvarez. Dirección Municipal de Salud de Guanabacoa. Vicedirección Docente. Calle Pepe Antonio esq. a Calixto García, Guanabacoa 11 100. La Habana, Cuba.

E-mail: rnara@infomed.sld.cu 